\title{
Assessment of Functional EST-SSR Markers (Sugarcane) in Cross-Species Transferability, Genetic Diversity among Poaceae Plants, and Bulk Segregation Analysis
}

\author{
Shamshad Ul Haq, ${ }^{1,2,3}$ Pradeep Kumar, ${ }^{1,4}$ R. K. Singh, ${ }^{1}$ Kumar Sambhav Verma, \\ Ritika Bhatt, ${ }^{2,3}$ Meenakshi Sharma, ${ }^{3}$ Sumita Kachhwaha, ${ }^{3}$ and S. L. Kothari ${ }^{2,3,5}$ \\ ${ }^{1}$ Biotechnology Division, UP Council of Sugarcane Research, Shahjahanpur 242001, India \\ ${ }^{2}$ Interdisciplinary Programme of Life Science for Advance Research and Education, University of Rajasthan, Jaipur 302004, India \\ ${ }^{3}$ Department of Botany, University of Rajasthan, Jaipur 302015, India \\ ${ }^{4}$ School of Biotechnology, Yeungnam University, Gyeongsan 712-749, Republic of Korea \\ ${ }^{5}$ Amity Institute of Biotechnology, Amity University Rajasthan, Jaipur 302006, India
}

Correspondence should be addressed to Shamshad Ul Haq; shamshadbiotech@gmail.com

Received 26 November 2015; Revised 13 April 2016; Accepted 26 April 2016

Academic Editor: Norman A. Doggett

Copyright (C) 2016 Shamshad Ul Haq et al. This is an open access article distributed under the Creative Commons Attribution License, which permits unrestricted use, distribution, and reproduction in any medium, provided the original work is properly cited.

\begin{abstract}
Expressed sequence tags (ESTs) are important resource for gene discovery, gene expression and its regulation, molecular marker development, and comparative genomics. We procured 10000 ESTs and analyzed 267 EST-SSRs markers through computational approach. The average density was one SSR $/ 10.45 \mathrm{~kb}$ or $6.4 \%$ frequency, wherein trinucleotide repeats $(66.74 \%)$ were the most abundant followed by di- $(26.10 \%)$, tetra- $(4.67 \%)$, penta- $(1.5 \%)$, and hexanucleotide $(1.2 \%)$ repeats. Functional annotations were done and after-effect newly developed 63 EST-SSRs were used for cross transferability, genetic diversity, and bulk segregation analysis (BSA). Out of 63 EST-SSRs, 42 markers were identified owing to their expansion genetics across 20 different plants which amplified 519 alleles at 180 loci with an average of 2.88 alleles/locus and the polymorphic information content (PIC) ranged from 0.51 to 0.93 with an average of 0.83 . The cross transferability ranged from $25 \%$ for wheat to $97.22 \%$ for Schlerostachya, with an average of $55.86 \%$, and genetic relationships were established based on diversification among them. Moreover, 10 EST-SSRs were recognized as important markers between bulks of pooled DNA of sugarcane cultivars through BSA. This study highlights the employability of the markers in transferability, genetic diversity in grass species, and distinguished sugarcane bulks.
\end{abstract}

\section{Introduction}

Sugarcane is a bioenergy crop belonging to the genus Saccharum L. of the tribe Andropogoneae (family: Poaceae). This tribe comprises grass species which have high economic value. The noble sugarcane varieties are developed from interspecific hybridization of Saccharum officinarum L. $(2 n=80)$ which has high sugar content with less disease tolerance and Saccharum spontaneum $(2 n=40$ to 120$)$ which provides stress, disease tolerance, and high fiber content for biomass. The taxonomy and genetic constitution of sugarcane are complicated due to complex interspecific aneupolyploid genome which makes chromosome numbers range from 100 to 130 [1]. Moreover, six Saccharum spp. (S. spontaneum, S. officinarum, S. robustum, S. edule, S. barberi, and S. sinense) and four Saccharum related genera (Erianthus, Miscanthus, Sclerostachya, and Narenga) have purportedly undergone interbreeding, forming the "Saccharum complex" $[2,3]$. The interbreeding has made their genome more complex and added to multigenic and/or multiallelic nature for most agronomic traits that made sugarcane breeding a more difficult task [4].

A vast array of genomic tools has been developed which has opened new ways to define the genetic architecture of sugarcane and helped to explore its functional system [1, 5]. Among the molecular markers, microsatellites are most 
favored for a variety of genetic applications due to their multiallelic nature, high reproducibility, cross transferability, codominant inheritance, abundance, and extensive genome coverage [6-8]. Microsatellites or simple sequences repeats (SSRs) are monotonous repetitions of very short (one to six) nucleotide motifs, which occur as interspersed repetitive elements in all eukaryotic and prokaryotic genomes. However, transcribed regions of the genome also contain enormous range of microsatellites that correspond to genic microsatellites or EST-SSRs. Therefore, expressed sequence tags (ESTs) are the short transcribed portions and involved in the variety of metabolic functions. The presence of the microsatellites in genes as well as ESTs unveils the biological significance of SSR distribution, expansion, and contraction on the function of the genes themselves [9].

Presently, huge amounts of expressed sequence tags have been deposited in public database (NCBI). In silico approaches to retrieve EST sequences from NCBI and functional annotations provide more constructive EST-SSRs or gene-based SSR (genic SSRs) marker development besides own EST libraries development. This method of the EST-SSR markers development provides the easiest way to reduce cost, time, and labours along with more meaningful marker identifications [10]. The presence of microsatellites in the genic region is found to be more conserved due to which they possess high reproducibility and high interspecific/intraspecific transferability. Hence, EST-SSR could be used for polymorphism, genetic diversity, cross transferability, and comparative mapping in different plant species. Accordingly, several genetic studies were done on sugarcane using microsatellite markers to decipher polymorphism, cross transferability, genetic diversity, informative marker detection through bulk segregation analysis (BSA), and comparative genomics [8, 11-13]. The objective of the present study was to retrieve EST sequences for more informative EST-SSR development and their genetic assessment within and across the taxa through cross transferability, genetic relationships, and bulk segregation analysis.

\section{Materials and Methods}

2.1. EST Sequences Retrieving, ESTs Assembling, and Microsatellites Identification. Total 10000 EST sequences of the Saccharum spp. were downloaded in Fasta format from National Centre for Biotechnology Information (NCBI) for microsatellites deciphering. Further, ESTs assembling was carried out using CAP3 programme (http://mobyle.pasteur .fr/cgi-bin/portal.py\#forms::cap3) for minimization of sequences redundancy. Microsatellite identification was carried out using MISA software (http://pgrc.ipk-gatersleben.de/ misa/) and the criteria for SSR detection were 6, 4, 3, 3, and 3 repeat units for di-, tri-, tetra-, penta-, and hexanucleotides, respectively. SSR primer pairs (forward and reverse) were designed for the selected EST sequences having microsatellites using online web tool, batch primer 3 pipeline [14].

2.2. EST-SSR Sequences Annotation. Assessment of EST sequences having SSR was done through blastn/blastx analysis for homology search and against nonredundant (nr) protein at the NCBI. Furthermore, functional annotation pipeline was also run at online tool for gene ontology (GO) which was intended for different GO functional classes like biological process, cellular component, and molecular function [15].

\subsection{PCR Amplification and Electrophoresis. PCR reactions} were carried out in a total of $10 \mu \mathrm{L}$ volume containing $25 \mathrm{ng}$ template DNA, $1.0 \mu \mathrm{L}(10 \mathrm{pmol} / \mu \mathrm{L})$ of each forward and reverse primer, $100 \mathrm{mM}$ of dNTPs, $0.5 \mathrm{U}$ of Taq DNA polymerase, and $1.0 \mu \mathrm{L}$ of 10x PCR buffer with $2.5 \mathrm{mM}$ of $\mathrm{MgCl}_{2}$. Amplification was performed in a thermal cycler (Bio-Rad) in the following conditions: initial denaturation at $94^{\circ} \mathrm{C}$ for $5 \mathrm{~min}$ followed by 30 amplification cycles of denaturation for $1 \mathrm{~min}$ at $94^{\circ} \mathrm{C}$ followed by annealing temperature $\left(T_{a}\right)$ for $1 \mathrm{~min}$ and then extension for $2 \mathrm{~min}$ at $72^{\circ} \mathrm{C}$; final extension at $72^{\circ} \mathrm{C}$ for $7 \mathrm{~min}$ was allowed. The PCR conditions particularly the annealing temperatures (varying from $52^{\circ} \mathrm{C}$ to $58^{\circ} \mathrm{C}$ ) for each primer were standardized and amplified products were stored at $4^{\circ} \mathrm{C}$. The PCR products were analyzed on a $7 \%$ native PAGE in vertical gel electrophoresis unit (Bangalore Genei $^{\mathrm{TM}}$ ) using TBE buffer. The sizes of amplified fragments were estimated using 50 bp DNA ladder (Fermentas). Gels were documented using ethidium bromide $(\mathrm{EtBr})$ stained dye.

\subsection{Evaluation of Saccharum EST-SSR across the Taxa through} Cross Transferability. The cross transferability of Saccharum derived EST-SSR markers was evaluated among the 20 accessions comprising seven cereals (wheat, maize, barley, rice, pearl millet, oat, and Sorghum), four Saccharum related genera (Erianthus, Miscanthus, Narenga, and Sclerostachya), three Saccharum species (51NG56 (S. robustum), N58 (S. spontaneum), and two clones of S. officinarum (Bandjermasin Hitam and Gunjera)), and five Saccharum commercial cultivars (CoS 88230, CoS 92423, UP 9530, CoS 8436, and CoS 91230). All genotypes were collected from the Sugarcane Research Institute Farm, UPCSR, Shahjahanpur, India. Furthermore, genomic DNA from young juvenile, disease-free, immature leaves was isolated for each genotype using CTAB (cetyl trimethylammonium bromide) method [16]. Isolated DNA samples were treated with RNAase for $1 \mathrm{~h}$ at $37^{\circ} \mathrm{C}$ and purified by phenol extraction (25 phenol: 24 chloroform: 1 isoamyl alcohol, $\mathrm{v} / \mathrm{v} / \mathrm{v}$ ) followed by ethanol precipitation [17] and stored at $-80^{\circ} \mathrm{C}$. DNA was quantified on $0.8 \%$ agarose gel and the working concentration of $25 \mathrm{ng} / \mu \mathrm{L}$ was obtained by making final adjustment in $10 \mathrm{mM}$ TE buffer.

2.5. Genetic Diversity Analysis. The assessment of EST-SSRs in genetic diversity analysis was done among 20 plants belonging to distinct groups comprising cereals, Saccharum related genera, Saccharum species, and Saccharum cultivars. The allelic data of 63 EST-SSR primers were used to ascertain the genetic relationships between 20 genotypes by clustering analysis. Amplified bands were scored as binary data in the form of present (1) or absent (0). Dendrogram was constructed by neighbour-joining and Jaccard's algorithm using 
FreeTree and TreeView software [18, 19]. The polymorphic information content (PIC) values were calculated for each primer by using the online resource of PIC Calculator (http:// www.liv.ac.uk/ kempsj/pic.html).

\subsection{Informative Assessment of Functional EST-SSR Markers} between Bulks. Plant materials were used as F2 mapping population comprising 209 genotypes of the sugarcane cultivars which were developed from cross between CoS 91230 (Parent; CoS $775 \times$ Co 1148) with CoS 8436 (Parent; MS 68/47 × Co 1148) from September to March (2010-2011). Grouping of genotypes was done according to their stem diameter (contrasting high and low stem diameter genotypes) into two sets. DNA extractions were carried out from both sets and equal quantities of genomic DNA from 10 extreme high stem diameter and 10 extreme low stem diameter genotypes were pooled into two bulks. PCR amplification was done in both bulks with newly developed EST-SSR primers for informative markers identifications through bulk segregation analysis (BSA) [20].

\section{Results and Discussion}

3.1. Mining of Microsatellites in EST Sequences and SSRs Characterization. Total 10,000 EST sequences related to Saccharum spp. were examined from NCBI for the simple sequence repeat (SSR) identification and characterization using computational approach. Prior to the marker deciphering, sequence assembly was performed and $6201(4201 \mathrm{~kb})$ nonredundant sequences were detected comprising 1752 contigs and 4449 singlets, wherein 406 SSRs were identified with 360 perfect SSRs and 37 sequences containing more than 1 SSR and 30 SSRs in compound formation. Therefore, computational and experimental approach to ascertain microsatellites in EST libraries from public database (NCBI) turned to be very cost effective and reduces time and labour besides expense of own libraries development. EST-SSRs are a more preferable DNA marker in the variety of genetic analysis and found to be more conserved as present in the transcribed region of the genome. These were found to be more transferable across the taxonomic boundaries and could be evaluated as most informative markers for variety of genomics applications $[10,21]$. These are more adapted in plants comparative genetic analysis for gene identification, gene mapping, marker-assisted-selection, transferability, and genetic diversity [7, 22-24]. Also, a variety of studies have been reported on sugarcane using EST-SSR markers for desired genetic analysis $[8,13,25,26]$.

The frequency of SSR in EST sequences was $6.4 \%$ including all the repeats except mononucleotide repeats. This result is comparatively higher compared to previous studies on sugarcane [8, 27-29]. Contrary to this, Singh et al. [13] reported higher frequency (9.3\%) in sugarcane. Kumpatla and Mukhopadhyay [30] also observed high range $(2.65 \%$ to $10.62 \%)$ of SSR frequency in different plant species. In general, about $5 \%$ of ESTs contained SSR which has been reported in many plant species [31]. These variations in microsatellite frequency could be attributed to the "search criteria" used, type of SSR motif, size of sequence data, and the mining tools used $[24,32]$. In other words, the density of the microsatellites was one SSR per $10.45 \mathrm{~kb}$ which is closely comparable to earlier studies in sugarcane with densities $1 \mathrm{SSR} / 10.9 \mathrm{~kb}$ [8] and $1 / 9 \mathrm{~kb}$ SSR [13].

Analysis revealed that trinucleotide repeats (66.74\%) were found to be more frequent followed by di- $(26.10 \%)$, tetra- $(4.67 \%)$, penta- $(1.5 \%)$, and hexanucleotide $(1.2 \%)$ repeats. Our observation of high frequency of trinucleotide repeats is in agreement with previous reports on sugarcane $[8,13,27-29,33]$. Several other studies have also represented high frequency of trinucleotide repeats in different plant species $[24,31,34-36]$. A total of 33 different types of motifs were identified of which four belonged to dinucleotide, eight belonged to trinucleotides, twelve belonged to tetranucleotide, five belonged to pentanucleotide, and two belonged to hexanucleotide repeats (Figure 1). We observed that motifs AG/CT and AT/AT were more frequent in dinucleotide repeat followed by motifs CCG/CGG, AGC/CTG, AGG/CCT, and ACG/CGT in trinucleotide repeat, motif AAAG/CTTT in tetranucleotide repeats, motif ACAGG/CCTGT in pentanucleotide repeats, and AACACC/GGTGTT in hexanucleotide repeats. The presence of motif CCG/CGG was also observed in sugarcane by different authors [13, 27]. Kantety et al. [37] also reported CCG/CGG motif as most abundant in wheat and Sorghum. Similarly, both Lawson and Zhang [38] and Da Maia et al. [39] also observed abundance of motif CCG/CGG in different member of the grass family. Victoria et al. [35] also decoded motif CCG/CGG in the lower plants (C. reinhardtii and $P$. patens). Thus, this predominance of CCG/CGG motif frequency has been related to a high GC-content [5]. Some motifs which are responsible for making unusual DNA folding structure (hairpin formed, bipartite triplex formed, and simple loop folding) also have effect on gene expressions and regulations mechanism, namely, CCT/AGG, CCG/GGC, GGA/TTC, and GAA/TTC motifs [40, 41]. Moreover, the presence of trinucleotide repeats in the coding region formed a distinct group and encoded amino acid tracts within the peptide [42]. We also observed predictable twenty different types of amino acids including stop codon. Alanine, arginine, glycine, proline, and serine were most frequent (Figure 2). This is in agreement with previous studies that reported on different plant species [11, 35, 43].

\subsection{Expressed Sequence Tags Annotation and Primers} Development. All EST sequences having SSRs were examined by functional annotation (blastn, blastx, and gene ontology). After-effect, sixty-three ESTs having SSRs were successfully identified on the basis of their involvement in the various metabolic processes (Figure 3). After-effect, sixty-three EST-SSRs primer pairs were designed for polymorphic nature, cross transferability, bulk segregation analysis, and genetic diversity in the test plants (Table 1). These selected EST-SSRs comprised all types of repeat motifs (excluding mononucleotide repeat), and among trinucleotide repeats they were highly frequent with GCT/CGA, TCC/AGG, and GGT/CCA repeat motifs. Similarly, Sharma et al. [44] also used functional annotation pipelines for the more prominent molecular markers development related to gene 
TABLE 1: Details of selected 63 EST-SSR primer pairs used for cross transferability, genetic diversity, and bulks segregation analysis.

\begin{tabular}{|c|c|c|c|c|c|c|c|}
\hline $\begin{array}{l}\text { Serial } \\
\text { number }\end{array}$ & Type & Primer sequence & $\begin{array}{l}\text { Annealing } \\
\text { temperature }\end{array}$ & SSR motif & PIC value & $E$-value & $\begin{array}{l}\text { Putative identities } \\
\text { (blastn/blastx) }\end{array}$ \\
\hline SYMS28 & $\mathrm{F}$ & GCGTCAGAGTGTTAAAACAAG & 53 & $(\mathrm{GCT})_{4}$ & 0.81 & $9.39 E-42$ & $\begin{array}{l}\text { Protein transport protein } \\
\text { Sec61 beta }\end{array}$ \\
\hline SYMS28 & $\mathrm{R}$ & GTGTAGAACTGGAGCATTGAG & & & & & \\
\hline SYMS29 & $\mathrm{F}$ & GGGCAAGCAAGAAACCAC & 52 & $(\mathrm{TCC})_{4}$ & 0.91 & $1.62 E-24$ & $\begin{array}{l}\text { Protein translation factor } \\
\text { SUI1 }\end{array}$ \\
\hline SYMS29 & $\mathrm{R}$ & GAAGAGGTCAACCAAGAACTC & & & & & \\
\hline SYMS30 & $\mathrm{F}$ & GCGTCAGAGTGTTAAAACAAG & 53 & $(\mathrm{GCT})_{4}$ & 0.86 & $1.00 E-21$ & Preprotein translocase Sec \\
\hline SYMS30 & $\mathrm{R}$ & GTGTAGAACTGGAGCATTGAG & & & & & \\
\hline SYMS31 & $\mathrm{F}$ & GAAGCTCCCAAGCTGCTA & 53 & $(\mathrm{AGCT})_{3}$ & 0.76 & $2.00 E-12$ & $\begin{array}{l}\text { Predicted: uncharacterized } \\
\text { protein }\end{array}$ \\
\hline SYMS31 & $\mathrm{R}$ & CCTACAGGAAAGATTTTAGGG & & & & & \\
\hline SYMS32 & $\mathrm{F}$ & GTCTCTTCTCCAGTTCTCCTT & 55 & $(\mathrm{TGCG})_{4}$ & 0.84 & $2.46 E-63$ & $\begin{array}{l}\text { Predicted: } \\
\text { actin-depolymerizing } \\
\text { factor }\end{array}$ \\
\hline SYMS32 & $\mathrm{R}$ & GCTCAACAAATGTCTCССТА & & & & & \\
\hline SYMS33 & $\mathrm{F}$ & TGCACTAACATGGTTGATGT & 54 & $(\mathrm{GAAG})_{3}$ & 0.86 & $2.82 E-90$ & $\begin{array}{l}\text { Hypothetical protein } \\
\text { SORBIDRAFT_03g046450 }\end{array}$ \\
\hline SYMS33 & $\mathrm{R}$ & GGTGATTGTAAGGGTCATCTT & & & & & \\
\hline SYMS34 & $\mathrm{F}$ & GTTAATGGTGGTTCCGTTC & 53 & $(\mathrm{GGC})_{6}$ & 0.88 & $4 E-20$ & $\begin{array}{l}\text { Predicted: uncharacterized } \\
\text { protein LOC101783547 }\end{array}$ \\
\hline SYMS34 & $\mathrm{R}$ & ATTATCAGCGCAGAGACATC & & & & & \\
\hline SYMS35 & $\mathrm{F}$ & GCGTCAGAGTGTTAAAACAAG & 52 & $(\mathrm{GCT})_{4}$ & 0.75 & $1.00 E-21$ & Preprotein translocase \\
\hline SYMS35 & $\mathrm{R}$ & GTGTAGAACTGGAGCATTGAG & & & & & \\
\hline SYMS36 & $\mathrm{F}$ & GGACTGTACAAGGACGACAG & 53 & $(\mathrm{GCT})_{4}$ & 0.70 & $1.14 E-41$ & $\begin{array}{l}\text { Protein transport protein } \\
\text { Sec61 beta subunit }\end{array}$ \\
\hline SYMS36 & $\mathrm{R}$ & TCTGCTTTCTTGGATATGGTA & & & & & \\
\hline SYMS37 & $\mathrm{F}$ & AAGAAGGATGCAAAGAAGAAG & 54 & $(\mathrm{GAT})_{4}$ & 0.90 & $3.08 E-81$ & $\begin{array}{l}\text { Hypothetical protein } \\
\text { SORBIDRAFT_03g046450 }\end{array}$ \\
\hline SYMS37 & $\mathrm{R}$ & AGGCTTAGTAACAGCAGGTTT & & & & & \\
\hline SYMS38 & $\mathrm{F}$ & AAGAAGGATGCAAAGAAGAAG & 56 & $(\mathrm{AGA})_{4}$ & 0.86 & $9.00 E-37$ & Hypothetical protein \\
\hline SYMS38 & $\mathrm{R}$ & AGGCTTAGTAACAGCAGGTTT & & & & & \\
\hline SYMS39 & $\mathrm{F}$ & GGACTGTACAAGGACGACAG & - & $(\mathrm{GCT})_{4}$ & - & $1.14 E-41$ & Protein transport protein \\
\hline SYMS39 & $\mathrm{R}$ & TCTGCTTTCTTGGATATGGTA & & & & & \\
\hline SYMS40 & $\mathrm{F}$ & GGACTGTACAAGGACGACAG & - & $(\mathrm{GCT})_{4}$ & - & $1.25 E-40$ & Preprotein translocase \\
\hline SYMS40 & $\mathrm{R}$ & TCTGCTTTCTTGGATATGGTA & & & & & \\
\hline SYMS41 & $\mathrm{F}$ & GGACTGTACAAGGACGACAG & - & $(\mathrm{GCT})_{4}$ & - & $9.62 E-42$ & $\begin{array}{l}\text { Protein transport protein } \\
\text { Sec61 beta subunit }\end{array}$ \\
\hline SYMS41 & $\mathrm{R}$ & TCTGCTTTCTTGGATATGGTA & & & & & \\
\hline SYMS42 & $\mathrm{F}$ & CCAAAGAGATCTTGCAGACTA & - & $(\mathrm{ATG})_{4}$ & - & $1.78 E-53$ & Jasmonate-induced protein \\
\hline SYMS42 & $\mathrm{R}$ & CCCAACACAACAACCAAT & & & & & \\
\hline SYMS43 & $\mathrm{F}$ & CCACACAAGCAAGAAATAAAC & - & $(\mathrm{GGT})_{4}$ & - & $8.57 E-74$ & Dirigent-like protein \\
\hline SYMS43 & $\mathrm{R}$ & TCGAACACTATGGTAAAGGTG & & & & & \\
\hline SYMS44 & $\mathrm{F}$ & GGACTGTACAAGGACGACAG & - & $(\mathrm{GCT})_{4}$ & - & $1.15 E-41$ & $\begin{array}{l}\text { Homeodomain-like } \\
\text { transcription factor }\end{array}$ \\
\hline SYMS44 & $\mathrm{R}$ & TCTGCTTTCTTGGATATGGTA & & & & & \\
\hline SYMS45 & $\mathrm{F}$ & GCGTCAGAGTGTTAAAACAAG & 53 & $(\mathrm{GCT})_{4}$ & 0.69 & $9.86 E-42$ & Protein transport protein \\
\hline
\end{tabular}


TABle 1: Continued.

\begin{tabular}{|c|c|c|c|c|c|c|c|}
\hline $\begin{array}{l}\text { Serial } \\
\text { number }\end{array}$ & Type & Primer sequence & $\begin{array}{l}\text { Annealing } \\
\text { temperature }\end{array}$ & SSR motif & PIC value & $E$-value & $\begin{array}{l}\text { Putative identities } \\
\text { (blastn/blastx) }\end{array}$ \\
\hline SYMS45 & $\mathrm{R}$ & GACTCTGCTTTCTTGGATATG & & & & & \\
\hline SYMS46 & $\mathrm{F}$ & AGCTATCTTTAGTGGGGACAT & 52 & $(\mathrm{CGT})_{4}$ & 0.90 & $1.82 E-44$ & $\begin{array}{l}\text { Hypothetical protein } \\
\text { SORBIDRAFT_09g006220 }\end{array}$ \\
\hline SYMS46 & $\mathrm{R}$ & GAGGTCTCATCGGAGCTTA & & & & & \\
\hline SYMS47 & $\mathrm{F}$ & AGGTCGTTTTAATTCCTTCC & 53 & $(\mathrm{GTTTT})_{3}$ & 0.77 & $1.00 E-21$ & Preprotein translocase Sec \\
\hline SYMS47 & $\mathrm{R}$ & CGTAAATATGAACGAGGTCAG & & & & & \\
\hline SYMS48 & $\mathrm{F}$ & AGGTCGTTTTAATTCCTTCC & 53 & $\left(\mathrm{TTTA}_{6}\right.$ & 0.90 & $4.00 E-20$ & TPA: hypothetical protein \\
\hline SYMS48 & $\mathrm{R}$ & CGTAAATATGAACGAGGTCAG & & & & & \\
\hline SYMS49 & $\mathrm{F}$ & GGACTGTACAAGGACGACAG & - & $(\mathrm{GCT})_{4}$ & - & $1.15 E-41$ & $\begin{array}{l}\text { Zinc finger A20 and AN1 } \\
\text { domains-containing } \\
\text { protein }\end{array}$ \\
\hline SYMS49 & $\mathrm{R}$ & TCTGCTTTCTTGGATATGGTA & & & & & \\
\hline SYMS50 & $\mathrm{F}$ & TCCAAGGATTTAGCTATGGAT & - & $(\mathrm{TGT})_{10}$ & - & $6.79 E-13$ & $\begin{array}{l}\text { TPA: seed maturation } \\
\text { protein }\end{array}$ \\
\hline SYMS50 & $\mathrm{R}$ & TTCAACTACACССТTCTGTTG & & & & & \\
\hline SYMS51 & $\mathrm{F}$ & GCGTCAGAGTGTTAAAACAAG & - & $(\mathrm{GCT})_{4}$ & - & $1.22 E-41$ & Hypothetical protein \\
\hline SYMS51 & $\mathrm{R}$ & ATTGTCACTTGCTATCCATTT & & & & & \\
\hline SYMS52 & $\mathrm{F}$ & САССТТСТТТССТТСТССТС & - & $(\mathrm{CGC})_{4}$ & - & $3.32 E-47$ & $\begin{array}{l}\text { V-type proton ATPase } \\
16 \mathrm{kDa} \text { proteolipid subunit }\end{array}$ \\
\hline SYMS52 & $\mathrm{R}$ & GTAGATACCGAGCACACCAG & & & & & \\
\hline SYMS53 & $\mathrm{F}$ & TCAGTTCAGGGATGACAATAG & 56 & $(\mathrm{CCGTGG})_{3}$ & 0.87 & $2.59 E-78$ & $\begin{array}{l}\text { Homeodomain-like } \\
\text { transcription factor } \\
\text { superfamily protein }\end{array}$ \\
\hline SYMS53 & $\mathrm{R}$ & GGATAGACTGAAATCTGCTCA & & & & & \\
\hline SYMS54 & $\mathrm{F}$ & CAACTCGACTCTTTTCTCTCA & - & $(\mathrm{CTC})_{5}$ & - & $4.13 E-08$ & $\begin{array}{l}\text { Protein transport protein } \\
\text { SEC31 }\end{array}$ \\
\hline SYMS54 & $\mathrm{R}$ & GGAGGTGGAACTTCCTGA & & & & & \\
\hline SYMS55 & $\mathrm{F}$ & GGACTGTACAAGGACGACAG & - & $(\mathrm{GCT})_{4}$ & - & $1.12 E-41$ & $\begin{array}{l}\text { Protein transport protein } \\
\text { Sec61 subunit beta-like } \\
\text { isoform }\end{array}$ \\
\hline SYMS55 & $\mathrm{R}$ & TCTGCTTTCTTGGATATGGTA & & & & & \\
\hline SYMS56 & $\mathrm{F}$ & GGACTGTACAAGGACGACAG & - & $(\mathrm{GCT})_{4}$ & - & $8.01 E-42$ & $\begin{array}{l}\text { Protein transport protein } \\
\text { Sec61 subunit beta-like } \\
\text { isoform }\end{array}$ \\
\hline SYMS56 & $\mathrm{R}$ & TCTGCTTTCTTGGATATGGTA & & & & & \\
\hline SYMS57 & $\mathrm{F}$ & AAACGATCAGATACCGTTGTA & - & $(\mathrm{CG})_{6}$ & - & $7.84 E-27$ & Caltractin \\
\hline SYMS57 & $\mathrm{R}$ & ATCAAAGAGATCAAAGGCTTC & & & & & \\
\hline SYMS58 & $\mathrm{F}$ & CATTTCGAAGCTCCTCCT & 52 & $(\mathrm{CCTCCG})_{6}$ & 0.74 & $5.97 E-66$ & $\begin{array}{l}\text { Zinc finger A20 and AN1 } \\
\text { domains-containing } \\
\text { protein }\end{array}$ \\
\hline SYMS58 & $\mathrm{R}$ & TAGGCTGCACAACAATAGTCT & & & & & \\
\hline SYMS59 & $\mathrm{F}$ & СТСССССАТТТСТСТТСС & 53 & $(\mathrm{GCAGCC})_{6}$ & 0.80 & $4.02 E-65$ & $\begin{array}{l}\text { Predicted: reticulon-like } \\
\text { protein } \mathrm{B} 1\end{array}$ \\
\hline SYMS59 & $\mathrm{R}$ & CAAGTACTCCAGCAGAGATGT & & & & & \\
\hline SYMS60 & $\mathrm{F}$ & СТТТТСССТСТТССТСТСТС & - & $(\mathrm{CCG})_{5}$ & - & $1.24 E-45$ & $\begin{array}{l}\text { Predicted: uncharacterized } \\
\text { tRNA-binding protein }\end{array}$ \\
\hline SYMS60 & $\mathrm{R}$ & TGTCACTAACACGAATCACAA & & & & & \\
\hline SYMS61 & $\mathrm{F}$ & СССТСТСССТGСТСТTТС & 54 & $(\mathrm{TCC})_{5}$ & 0.79 & $4.14 E-57$ & $\begin{array}{l}\text { Actin-depolymerizing } \\
\text { factor } 3\end{array}$ \\
\hline
\end{tabular}


TABLE 1: Continued.

\begin{tabular}{|c|c|c|c|c|c|c|c|}
\hline $\begin{array}{l}\text { Serial } \\
\text { number }\end{array}$ & Type & Primer sequence & $\begin{array}{l}\text { Annealing } \\
\text { temperature }\end{array}$ & SSR motif & PIC value & $E$-value & $\begin{array}{l}\text { Putative identities } \\
\text { (blastn/blastx) }\end{array}$ \\
\hline SYMS61 & $\mathrm{R}$ & CAGTCACAAAGTCGAAATCAT & & & & & \\
\hline SYMS62 & $\mathrm{F}$ & ACAACTCTTCAGTCTTCACGA & 54 & $(\mathrm{CAAC})_{3}$ & 0.85 & $4.40 E-66$ & $\begin{array}{l}\text { Truncated alcohol } \\
\text { dehydrogenase }\end{array}$ \\
\hline SYMS62 & $\mathrm{R}$ & CCAATCTTGACATCCTTGAC & & & & & \\
\hline SYMS63 & $\mathrm{F}$ & GCACGGTGAAGTTCTAGTTC & 54 & $(\text { TCGAT })_{4}$ & 0.67 & $3.11 E-31$ & $\begin{array}{l}\text { Hypothetical protein } \\
\text { SORBIDRAFT_08g002800 }\end{array}$ \\
\hline SYMS63 & $\mathrm{R}$ & CAGCTTCACTCATGAATTTTT & & & & & \\
\hline SYMS64 & $\mathrm{F}$ & GGACTGTACAAGGACGACAG & - & $(\mathrm{GCT})_{4}$ & - & $1.08 E-41$ & $\begin{array}{l}\text { Protein transport protein } \\
\text { Sec61 subunit beta-like } \\
\text { isoform }\end{array}$ \\
\hline SYMS64 & $\mathrm{R}$ & TCTGCTTTCTTGGATATGGTA & & & & & \\
\hline SYMS65 & $\mathrm{F}$ & AACACAAGCAAGAAATAAACG & 53 & $(\mathrm{GGT})_{4}$ & 0.51 & $3.42 E-74$ & Dirigent-like protein \\
\hline SYMS65 & $\mathrm{R}$ & AACACTATGGTCAAGGTGGTA & & & & & \\
\hline SYMS66 & $\mathrm{F}$ & GCGTCAGAGTGTTAAAACAAG & 52 & $(\mathrm{GCT})_{4}$ & 0.58 & $1.01 E-41$ & $\begin{array}{l}\text { Protein transport protein } \\
\text { Sec61 subunit beta-like } \\
\text { isoform }\end{array}$ \\
\hline SYMS66 & $\mathrm{R}$ & GAAATCGCTCTATAAGGTTCC & & & & & \\
\hline SYMS67 & $\mathrm{F}$ & TCTCTCTGAAGATGATGCTTT & 52 & $(\mathrm{AAG})_{5}$ & 0.90 & $4.25 E-83$ & $\begin{array}{l}\text { Hypothetical protein } \\
\text { SORBIDRAFT_03g005100 }\end{array}$ \\
\hline SYMS67 & $\mathrm{R}$ & GTTAAGAGGCTTCCAAAGAAC & & & & & \\
\hline SYMS68 & $\mathrm{F}$ & CAGCTCGTCGTCTTCTTTT & - & $(\mathrm{GTC})_{5}$ & & $2.00 E-55$ & $\begin{array}{l}\text { Putative } \\
\text { ubiquitin-conjugating } \\
\text { enzyme family }\end{array}$ \\
\hline SYMS68 & $\mathrm{R}$ & GTGGCTTGTTTGGATATTCTT & & & & & \\
\hline SYMS69 & $\mathrm{F}$ & GGACTGTACAAGGACGACAG & 54 & $(\mathrm{GCT})_{4}$ & 0.79 & $9.28 E-42$ & $\begin{array}{l}\text { Protein transport protein } \\
\text { Sec61 subunit beta-like } \\
\text { isoform }\end{array}$ \\
\hline SYMS69 & $\mathrm{R}$ & CGTCAGACGTACTGAAATGTT & & & & & \\
\hline SYMS70 & $\mathrm{F}$ & AACACAAGCAAGAAATAAACG & 53 & $(\mathrm{GGT})_{4}$ & 0.77 & $1.58 E-73$ & Putative dirigent protein \\
\hline SYMS70 & $\mathrm{R}$ & AACACTATGGTCAAGGTGGTA & & & & & \\
\hline SYMS71 & $\mathrm{F}$ & GGACTGTACAAGGACGACAG & - & $(\mathrm{GCT})_{4}$ & - & $9.86 E-42$ & $\begin{array}{l}\text { Protein transport protein } \\
\text { Sec61 subunit beta-like } \\
\text { isoform }\end{array}$ \\
\hline SYMS71 & $\mathrm{R}$ & TCTGCTTTCTTGGATATGGTA & & & & & \\
\hline SYMS72 & $\mathrm{F}$ & СССТСТСССТGСТСТТТС & 55 & $(\mathrm{TCC})_{4}$ & 0.89 & $4.36 E-57$ & $\begin{array}{l}\text { Actin-depolymerizing } \\
\text { factor } 3\end{array}$ \\
\hline SYMS72 & $\mathrm{R}$ & CAGTCACAAAGTCGAAATCAT & & & & & \\
\hline SYMS73 & $\mathrm{F}$ & GGACTGTACAAGGACGACAG & 55 & $(\mathrm{GCT})_{4}$ & 0.83 & $1.09 E-41$ & $\begin{array}{l}\text { Protein transport protein } \\
\text { Sec61 subunit beta-like } \\
\text { isoform }\end{array}$ \\
\hline SYMS73 & $\mathrm{R}$ & TCTGCTTTCTTGGATATGGTA & & & & & \\
\hline SYMS74 & $\mathrm{F}$ & GGACTGTACAAGGACGACAG & 52 & $(\mathrm{GCT})_{4}$ & 0.88 & $9.51 E-42$ & Preprotein translocase Sec \\
\hline SYMS74 & $\mathrm{R}$ & TCTGCTTTCTTGGATATGGTA & & & & & \\
\hline SYMS75 & $\mathrm{F}$ & GCACCCCCAATTCGAACG & 52 & $(\mathrm{ACG})_{3}$ & 0.93 & $1.78 E-68$ & $\begin{array}{l}\text { TPA: general regulatory } \\
\text { factor } 1\end{array}$ \\
\hline SYMS75 & $\mathrm{R}$ & CGGTAGTCCTTGATGAGTGT & & & & & \\
\hline SYMS76 & $\mathrm{F}$ & GGACTGTACAAGGACGACAG & 52 & $(\mathrm{GCT})_{4}$ & 0.78 & $4.79 E-41$ & $\begin{array}{l}\text { Protein transport protein } \\
\text { Sec61 subunit beta-like } \\
\text { isoform }\end{array}$ \\
\hline SYMS76 & $\mathrm{R}$ & TCTGCTTTCTTGGATATGGTA & & & & & \\
\hline SYMS77 & $\mathrm{F}$ & CACGCAACGCAAGCACAG & 55 & $(\mathrm{CCAT})_{3}$ & 0.93 & $8.34 E-70$ & $\begin{array}{l}\text { Hypothetical protein } \\
\text { SORBIDRAFT_10g030160 }\end{array}$ \\
\hline
\end{tabular}


TABle 1: Continued.

\begin{tabular}{|c|c|c|c|c|c|c|c|}
\hline $\begin{array}{l}\text { Serial } \\
\text { number }\end{array}$ & Type & Primer sequence & $\begin{array}{l}\text { Annealing } \\
\text { temperature }\end{array}$ & SSR motif & PIC value & $E$-value & $\begin{array}{l}\text { Putative identities } \\
\text { (blastn/blastx) }\end{array}$ \\
\hline SYMS77 & $\mathrm{R}$ & AAGTTGATTCACCCTCATTCT & & & & & \\
\hline SYMS78 & $\mathrm{F}$ & CACGCAACGCAAGCACAG & 53 & $(\mathrm{CGATC})_{3}$ & 0.92 & $1.04 E-41$ & $\begin{array}{l}\text { Translocon-associated } \\
\text { protein alpha subunit } \\
\text { precursor }\end{array}$ \\
\hline SYMS78 & $\mathrm{R}$ & AAGTTGATTCACCСTCATTCT & & & & & \\
\hline SYMS79 & $\mathrm{F}$ & GGACTGTACAAGGACGACAG & 53 & $(\mathrm{GCT})_{4}$ & 0.91 & $1.04 E-41$ & $\begin{array}{l}\text { Protein transport protein } \\
\text { Sec61 subunit beta-like } \\
\text { isoform }\end{array}$ \\
\hline SYMS79 & $\mathrm{R}$ & TCTGCTTTCTTGGATATGGTA & & & & & \\
\hline SYMS80 & $\mathrm{F}$ & CTTGATCCTTGACAAAAGAGA & 52 & $(\mathrm{AG})_{6}$ & 0.87 & $2.25 E-59$ & $\begin{array}{l}\text { Predicted: } \\
\text { ubiquitin-conjugating } \\
\text { enzyme E2 }\end{array}$ \\
\hline SYMS80 & $\mathrm{R}$ & ATTGCTGTTGATATTTGGATG & & & & & \\
\hline SYMS81 & $\mathrm{F}$ & GCGTCAGAGTGTTAAAACAAG & 53 & $(\mathrm{GCT})_{4}$ & 0.87 & $8.01 E-42$ & $\begin{array}{l}\text { Protein transport protein } \\
\text { Sec61 subunit beta-like } \\
\text { isoform }\end{array}$ \\
\hline SYMS81 & $\mathrm{R}$ & GTGTAGAACTGGAGCATTGAG & & & & & \\
\hline SYMS82 & $\mathrm{F}$ & TATCAACAAGCCTTCCATTC & 53 & $(\mathrm{GTG})_{4}$ & 0.90 & $1.12 E-30$ & $\begin{array}{l}\text { Glycine-rich RNA-binding } \\
\text { protein } 2\end{array}$ \\
\hline SYMS82 & $\mathrm{R}$ & GGCTATAGTCACCACGGTAG & & & & & \\
\hline SYMS83 & $\mathrm{F}$ & CGACAGGGAGAAGAGTACAG & 55 & $(\mathrm{GCT})_{4}$ & 0.87 & $9.39 E-42$ & $\begin{array}{l}\text { Protein transport protein } \\
\text { Sec61 subunit beta-like } \\
\text { isoform }\end{array}$ \\
\hline SYMS83 & $\mathrm{R}$ & GACTCTGCTTTCTTGGATATG & & & & & \\
\hline SYMS84 & $\mathrm{F}$ & GCGTCAGAGTGTTAAAACAAG & 53 & $(\mathrm{GCT})_{4}$ & 0.75 & $1.14 E-41$ & $\begin{array}{l}\text { Protein transport protein } \\
\text { Sec61 subunit beta-like } \\
\text { isoform }\end{array}$ \\
\hline SYMS84 & $\mathrm{R}$ & AATCGCTCTATAAGGTTCCTC & & & & & \\
\hline SYMS85 & $\mathrm{F}$ & СТСТТСТТСАССААТТССТСТ & - & $(\mathrm{CCG})_{6}$ & - & $1.14 E-51$ & $\begin{array}{l}\text { Protein transport protein } \\
\text { Sec61 subunit beta-like } \\
\text { isoform }\end{array}$ \\
\hline SYMS85 & $\mathrm{R}$ & CAAACCTCATAAAGAGTGCAG & & & & & \\
\hline SYMS86 & $\mathrm{F}$ & GGGCAAGCAAGAAACCAC & 54 & $(\mathrm{TCC})_{4}$ & 0.93 & $1.16 E-28$ & $\begin{array}{l}\text { TPA: translation initiation } \\
\text { factor } 1\end{array}$ \\
\hline SYMS86 & $\mathrm{R}$ & CGTACATGAACGTAGTCCTTT & & & & & \\
\hline SYMS87 & $\mathrm{F}$ & GCGTCAGAGTGTTAAAACAAG & - & $(\mathrm{GCT})_{4}$ & - & $1.19 E-41$ & $\begin{array}{l}\text { Protein transport protein } \\
\text { Sec61 beta subunit }\end{array}$ \\
\hline SYMS87 & $\mathrm{R}$ & AATCGCTCTATAAGGTTCCTC & & & & & \\
\hline SYMS88 & $\mathrm{F}$ & TTATAAGGAAATCCCCCACT & - & $(\mathrm{GCC})_{4}$ & - & $7.71 E-55$ & $\begin{array}{l}\text { Hypothetical protein } \\
\text { SORBIDRAFT_09g000970 }\end{array}$ \\
\hline SYMS88 & $\mathrm{R}$ & CACCAAGTACTCATCCATCAT & & & & & \\
\hline SYMS89 & $\mathrm{F}$ & CATCTCCTGCTAACAATTCAC & 55 & $(\mathrm{TGC})_{4}$ & 0.91 & $9.64 E-60$ & $\begin{array}{l}\text { Predicted: NAC } \\
\text { domain-containing protein }\end{array}$ \\
\hline SYMS89 & $\mathrm{R}$ & ATTTATAGGTTGGCACCAGAG & & & & & \\
\hline SYMS90 & $\mathrm{F}$ & GCGTCAGAGTGTTAAAACAAG & 53 & $(\mathrm{GCT})_{4}$ & 0.85 & $1.00 E-22$ & $\begin{array}{l}\text { Protein transport protein } \\
\text { Sec61 subunit beta-like } \\
\text { isoform }\end{array}$ \\
\hline SYMS90 & $\mathrm{R}$ & GTGTAGAACTGGAGCATTGAG & & & & & \\
\hline
\end{tabular}




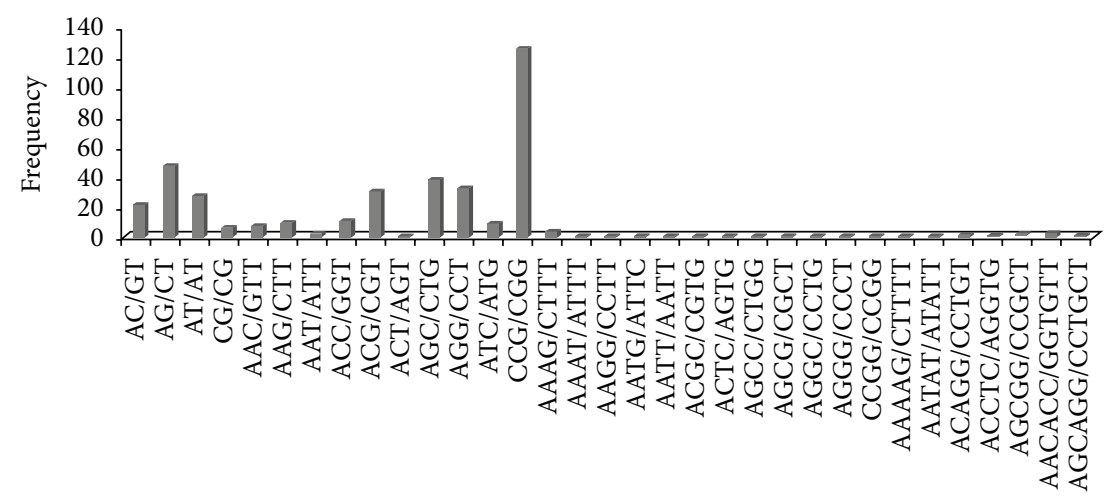

Types of motifs

FIGURE 1: Details of 33 different types of nucleotide repeat motifs belonging to di-, tri-, tetra-, penta-, and hexanucleotide repeat motifs with sequence complementarity.

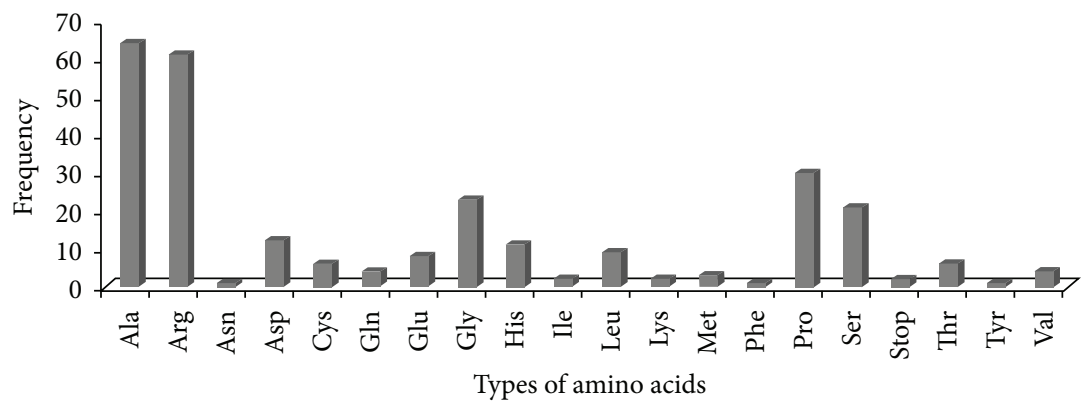

FIGURE 2: Details of different types of predicted amino acids encoded by trinucleotide repeat motifs.

transcripts. Selected EST-SSRs were associated with various pathways of metabolic process, namely, GO:0006281 DNA repair, GO:0006301 postreplication repair, GO:0016070 RNA metabolic process, GO:0016070 RNA metabolic process, GO:0006446 regulation of translational initiation, GO:0015991 ATP hydrolysis coupled proton transport, GO:0006629 lipid metabolic process, GO:0015031 protein transport, GO:0005667 transcription factor complex, GO:0005815 microtubule organizing centre, GO:0003743 translation initiation factor activity, GO:0017005 3'tyrosyl-DNA phosphodiesterase activity, GO:0030042 actin filament depolymerization, and GO:0015078 hydrogen ion transmembrane transporter activity, and so forth (see the complete details of the most promising hits of gene ontology of EST-SSRs in the supplementary table available online at http://dx.doi.org/10.1155/2016/7052323).

3.3. Assessment of EST-SSR Marker in Selected Plants. A set of 63 EST-SSR primers were evaluated for PCR optimization, polymorphism, and cross amplification in twenty genotypes belonging to cereals plants and Saccharum related genera and Saccharum species and their commercial cultivars, of which 42 EST-SSR primers produced successful amplifications with both expected and unexpected sizes (Figure 4). Among 42 EST-SSRs, twenty-eight belonged to trinucleotide repeats with then seven of tetra-, three of penta-, three of hexa-, and one of dinucleotide repeats. Meanwhile, PCR amplifications produced 519 alleles (expected size) at 180 loci with an average of 2.88 alleles per locus. This result is comparable with earlier studies that reported on various plant species, namely, 2.79 alleles/locus in rice varieties [45], 2.9 to 6.0 alleles per locus in maize [46], and 3.04 alleles/locus in rye [47]. However, our result of alleles per locus is lower compared to previous studies that reported on sugarcane, that is, 6.04 alleles/locus [28], 7.55 alleles/locus [29], and 6.0 alleles/locus [48]. The polymorphic information content (PIC) was extended from 0.51 to 0.93 with an average of 0.83 . It could be encompassed that low and high range of allelic amplifications with ESTSSRs correspond to marker polymorphism and low level of polymorphism from EST-SSRs might be due to possible selection against alterations in the conserved sequences of EST-SSRs $[49,50]$.

3.4. Cross Transferability. The potentials of EST-SSR primers were examined for cross transferability among 20 plant species belonging to cereals and Saccharum related genera and Saccharum species and their cultivars under the same PCR conditions. However, 42 EST-SSRs showed successful amplifications among all the selected plants. The cross transferability was estimated to be $27.22 \%$ in wheat, $27.22 \%$ in maize, $47.22 \%$ in barely, $46.66 \%$ in rice, $36.11 \%$ in pearl millet, $55.55 \%$ in oat, $26.11 \%$ in Sorghum, $88.33 \%$ in Narenga, $98.88 \%$ in Sclerostachya, $71.11 \%$ in Erianthus, $60.0 \%$ in Miscanthus, $73.33 \%$ in Bandjermasin Hitam, 55.55\% in Gunjera, $75.55 \%$ in 


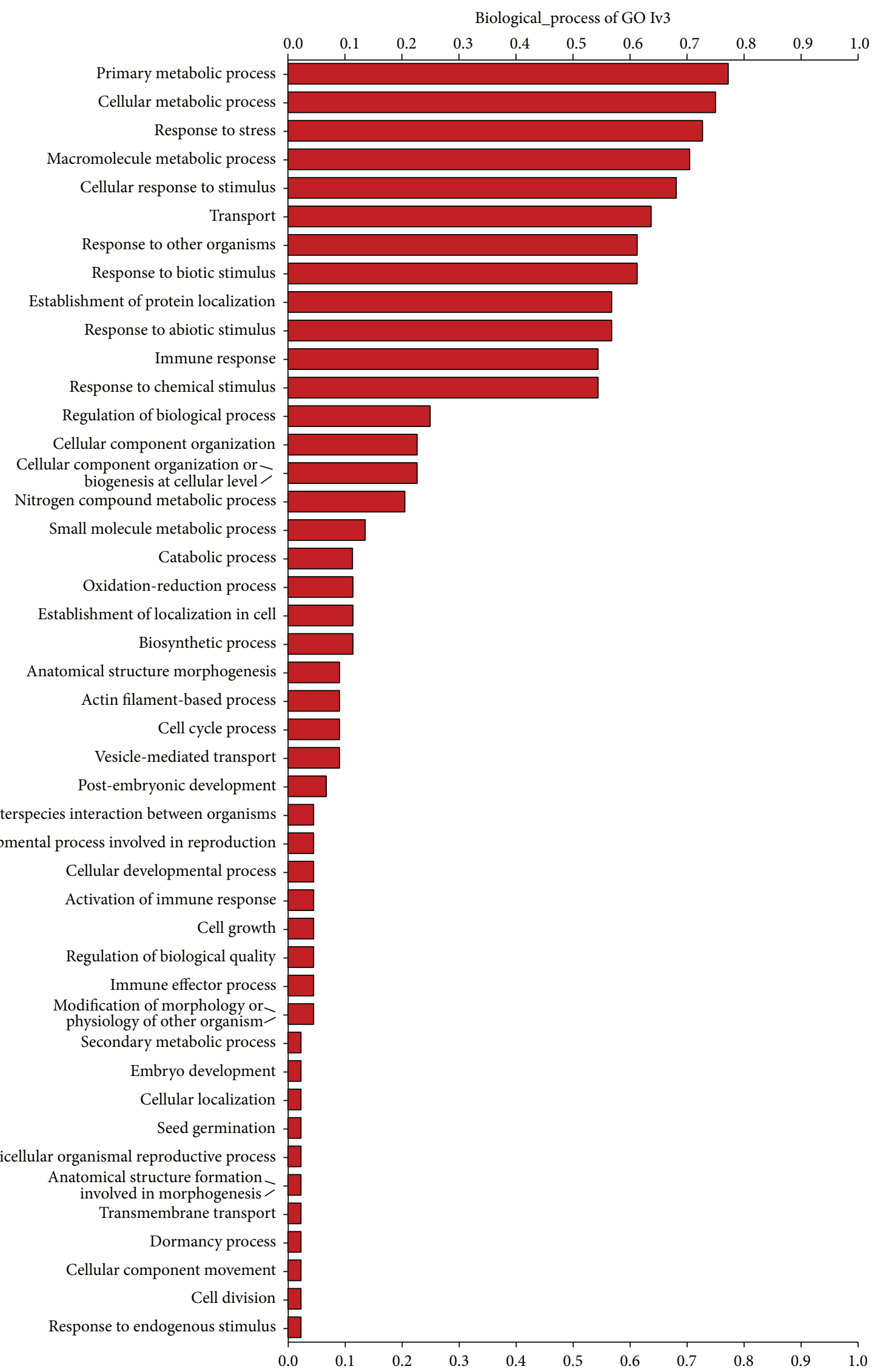

(a)

Figure 3: Continued. 


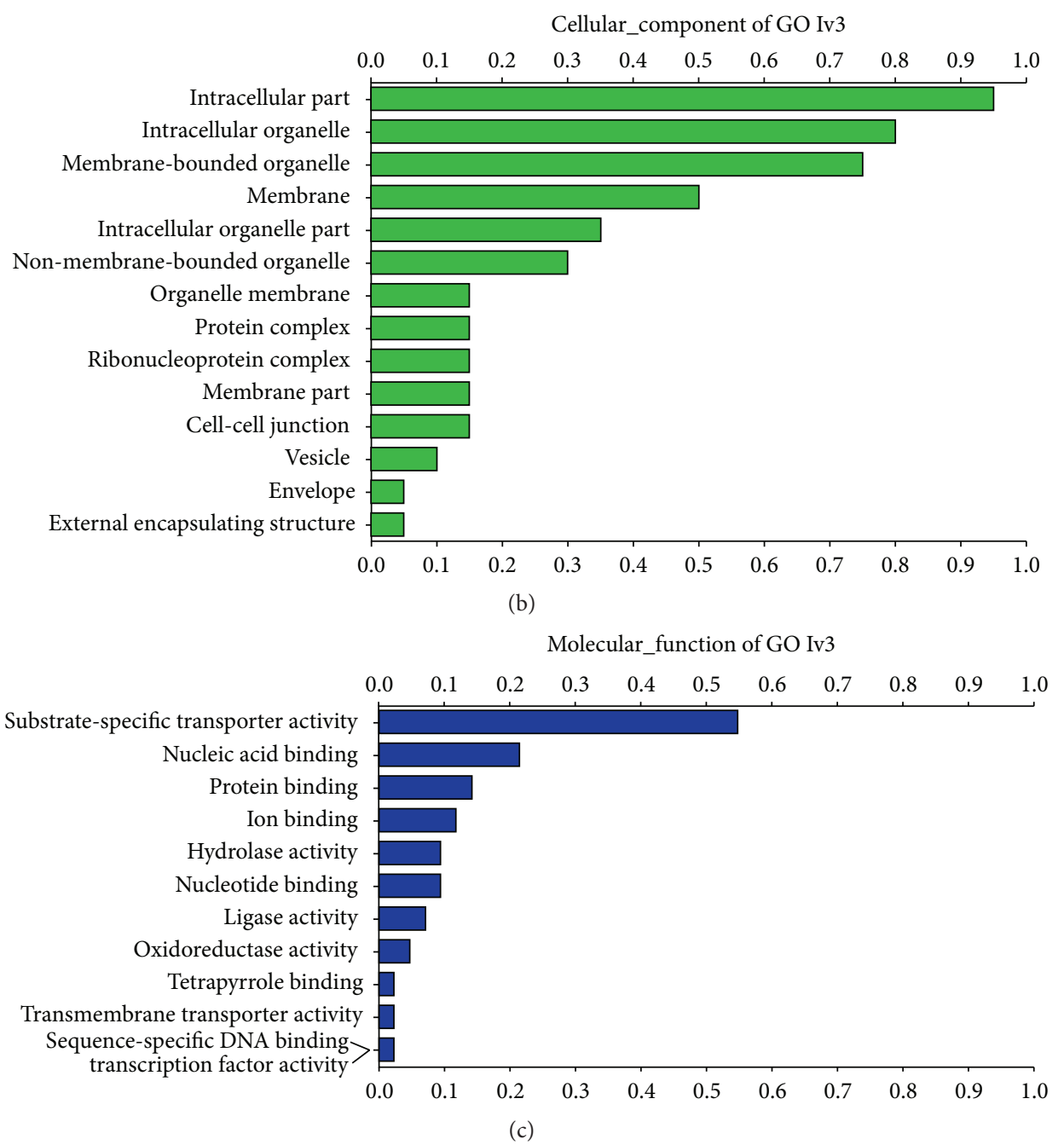

Figure 3: Most promising results of gene ontology (GO) as horizontal bar graphs. These graphs represent the distribution of GO terms categorized as a biological process (a), cellular component (b), and molecular function (c).

$51 N G 56,55.0 \%$ in N58, 50.56\% in CoS $92423,58.88 \%$ in CoS $88230,51.11 \%$ in UP 9530, 52.78\% in CoS 91230, and 60.0\% in CoS 8436. Meanwhile, the frequency distributions of cross transferability of EST-SSRs ranged from $26.11 \%$ for Sorghum to $98.88 \%$ for Sclerostachya, with an average of $55.86 \%$ (Table 2). Saccharum related genera (79.58\%) and Saccharum species $(64.86 \%)$ showed high rate of cross transferability compared to other groups. This is in agreement with previous studies reported on Saccharum species and Saccharum related genera $[12,13,51]$. Several earlier studies related to cross transferability have been reported on distinct plant groups from different families using EST-SSRs markers [7, 52, 53]. This suggests that transferring ability of genic markers makes it compatible to determine genetic studies across the taxa for utilization in mapping of genes from related species along with genera and identification of suspended hybridization. This can also aid vigilance of the introgression of genetic entity from wild relatives to cultivated, comparative mapping and establishing evolutionary relationship between them. Thus, microsatellites derived from expressed region of the genome are expected to be more conserved and more transferable across taxa.

3.5. Genetic Diversity Analysis by EST-SSRs. In order to evaluate the potential of EST-SSRs, the genetic analysis was done among 20 genotypes belonging to 7 cereals (wheat, maize, barley, rice, pearl millet, oat, and Sorghum), 4 Saccharum related genera (Erianthus, Miscanthus, Narenga, and Sclerostachya), 3 Saccharum species (51NG56 (S. robustum), N58 (S. spontaneum), and two of S. officinarum clones (Gunjera and Bandjermasin Hitam)), and 5 sugarcane commercial cultivars (CoS 8436, CoS 91230, CoS 88230, UP 9530, and $\operatorname{CoS}$ 92423). The generated allelic data were used for genetic relationships analysis by making dendrogram based on Jaccard's and neighbour-joining algorithm using FreeTree and TreeView software. The dendrogram fell into three major clusters with several edges, cluster I with eight genotypes comprising most of Saccharum species and their commercial cultivars, cluster II encompassing six genotypes of most of cereals species, and cluster III with six species comprising 


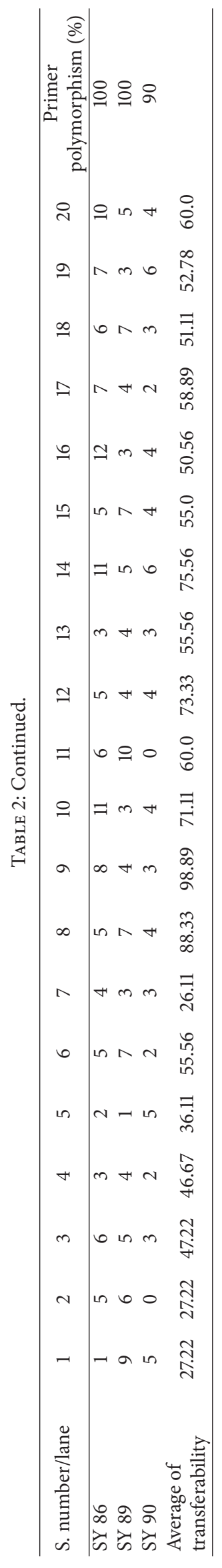




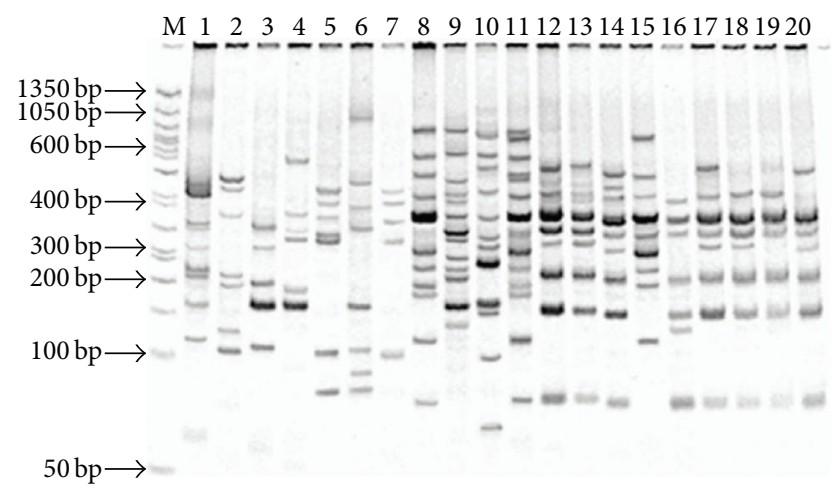

FIGURE 4: The gel represents PCR amplification profile with SYMS37 primer among twenty different plant species. Lanes: 1 wheat, 2 maize, 3 barley, 4 rice, 5 pearl millet, 6 oat, 7 Sorghum, 8 Narenga, 9 Schlerostachya, 10 Erianthus, 11 Miscanthus, 12 Bandjermasin Hitam, 13 Gunjera, 14 51NG56, 15 N58, $16 \operatorname{CoS} 92423,17 \operatorname{CoS} 88230,18$ UP 9530, $19 \operatorname{CoS} 91230$, and $20 \operatorname{CoS} 8436$.

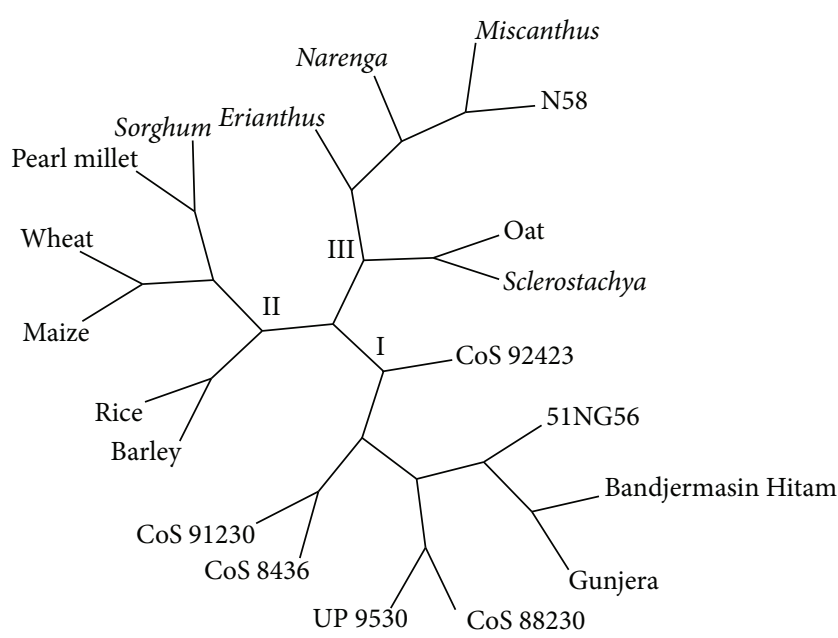

FIGURE 5: Dendrogram is constructed based on allelic data produced from 42 EST-SSR markers using FreeTree and TreeView software.

most of the Saccharum related genera along with some interventions (Figure 5). This relationship is in agreement with previous studies reported by other authors $[12,13,51,54]$. Our EST-SSRs markers showed close syntenic relationship and their evolutionary nature among the 20 genotypes into three major clusters with some genotypes divergence. These relationships have resulted from the expansion and contraction of SSRs in conserved EST sequences within the same group of plant species along with some variation having resulted from higher evolutionary divergence among them. Several earlier studies also reported on genetic diversity analysis within and across the plant taxa using molecular marker [7, 8, 24, 48, 52, 55-57]. Thus, microsatellite markers distinguished all the genotypes to certain extent and also provided the realistic estimate of genetic diversity among them.

3.6. Bulk Segregation Analysis (BSA) in Sugarcane. All the 42 EST-SSR markers were evaluated in pooled DNA bulks

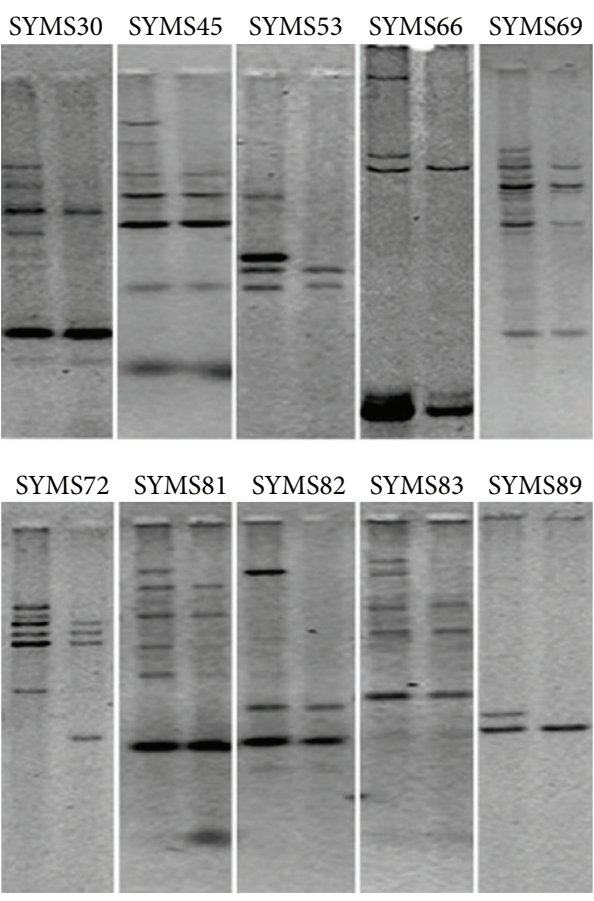

FIGURE 6: The gel represents polymorphism and discrimination between bulks of pooled DNA with contrasting high and low plant diameter through bulk segregation analysis.

of contrasting trait of sugarcane cultivars (CoS $91230(\mathrm{CoS}$ $775 \times$ Co 1148) cross with CoS 8436 (MS 68/47 × Co 1148)) for the identification of reporter EST-SSR markers based on their allelic differences between them. Interestingly, 10 markers showed polymorphic nature and apparently discriminating potential between bulks through bulk segregation analysis (Figure 6). Among these, markers SYMS30, SYMS53, SYMS82, and SYMS89 showed a better response to discriminating the bulks. BSA is the strategy that involves the identification of genetic markers associated with character or trait which are based on their allelic differences between bulks [20]. Earlier studies have been established in sugarcane for the most prominent molecular markers detection linked to desirable traits through BSA. For example, molecular markers apparently linked to high fiber content in Saccharum species [58-60] and molecular markers used for QTL analysis and utilized for generating genetic maps around resistance genes in sugarcane against diseases and pests through BSA [12, 61, 62]. Several other studies also reported on selection of different agronomic traits in sugarcane for breeding programme with the development of molecular markers through BSA [1, 63-65]. Alternatively, BSA approach has been recently used for various purposes against the identification of differential expressed gene associated with both qualitative and quantitative using of the cDNA-AFLP approach [66-69]. Thus, BSA approach provides the easiest way in the direction of trait linked marker identification and also makes it possible to select informative markers beside evaluations of each marker in the whole progeny. 


\section{Conclusion}

The present study was intended for identification and characterization of SSR in Saccharum spp. expressed sequence tag which is retrieved from public database (NCBI). Further, functional annotation was feasible to identify the most eminent EST-SSR markers selection. Therefore, this is the bypass way for EST-SSR markers development which reduces cost and time and provides an efficient way to analyze the transcribed portion of genome besides expense of own libraries development. A total of 63 EST-SSR markers were developed and experimentally validated for cross transferability along with their genetic relationships and also used for differentiation between pooled DNA bulks of Saccharum cultivars. These markers showed successful transferability rate among the twenty genotypes and established genetic diversity among cereals, Saccharum species/cultivars, and Saccharum related genera with some inconsistency. Further, some prominent marker also distinguished pooled DNA bulks of sugarcane cultivars based on stem diameter. Consequently, these ESTSSR markers were found to be more convenient which made it easy for us to use them as informative markers in further genetic studies in sugarcane breeding programme.

\section{Competing Interests}

The authors declare that there is no conflict of interests regarding the publication of this paper.

\section{Acknowledgments}

Authors are highly grateful to the Division of Biotechnology, UP Council of Sugarcane Research, for providing an opportunity and facilities for research works. Authors are also grateful to Director, UP Council of Sugarcane Research, Shahjahanpur, UP, India for their moral support. Authors also acknowledge University of Rajasthan for providing DBTIPLS and DBT-BIF facilities.

\section{References}

[1] J. Y. Hoarau, G. Souza, A. D’Hont et al., Sugarcane, A Tropical Crop with a Highly Complex Genome, Plant Genomics, Sciences Publishers, Enfield, NH, USA, 2006.

[2] B. Roach and J. Daniels, "A review of the origin and improvement of sugarcane," Copersucar International Sugarcane Breeding Workshop, vol. 1, pp. 1-31, 1987.

[3] A. D’Hont, D. Ison, K. Alix, C. Roux, and J. C. Glaszmann, "Determination of basic chromosome numbers in the genus Saccharum by physical mapping of ribosomal RNA genes," Genome, vol. 41, no. 2, pp. 221-225, 1998.

[4] R. E. Casu, J. M. Manners, G. D. Bonnett et al., "Genomics approaches for the identification of genes determining important traits in sugarcane," Field Crops Research, vol. 92, no. 2-3, pp. 137-147, 2005.

[5] M. Morgante and A. M. Olivieri, "PCR-amplified microsatellites as markers in plant genetics," Plant Journal, vol. 3, no. 1, pp. 175$182,1993$.
[6] M. Agarwal, N. Shrivastava, and H. Padh, "Advances in molecular marker techniques and their applications in plant sciences," Plant Cell Reports, vol. 27, no. 4, pp. 617-631, 2008.

[7] S. U. Haq, R. Jain, M. Sharma, S. Kachhwaha, and S. L. Kothari, "Identification and characterization of microsatellites in expressed sequence tags and their cross transferability in different plants," International Journal of Genomics, vol. 2014, Article ID 863948, 12 pages, 2014.

[8] S. K. Parida, A. Pandit, K. Gaikwad et al., "Functionally relevant microsatellites in sugarcane unigenes," BMC Plant Biology, vol. 10, no. 1, article 251, 2010.

[9] X. Li, W. Gao, H. Guo, X. Zhang, D. D. Fang, and Z. Lin, "Development of EST-based SNP and InDel markers and their utilization in tetraploid cotton genetic mapping," $B M C$ Genomics, vol. 15, no. 1, article 1046, 2014.

[10] Q. Kong, C. Xiang, Z. Yu et al., "Mining and charactering microsatellites in Cucumis melo expressed sequence tags from sequence database," Molecular Ecology Notes, vol. 7, no. 2, pp. 281-283, 2007.

[11] S. Gupta, K. P. Tripathi, S. Roy, and A. Sharma, "Analysis of unigene derived microsatellite markers in family solanaceae," Bioinformation, vol. 5, no. 3, pp. 113-121, 2010.

[12] R. K. Singh, R. B. Singh, S. P. Singh, and M. L. Sharma, "Identification of sugarcane microsatellites associated to sugar content in sugarcane and transferability to other cereal genomes," Euphytica, vol. 182, no. 3, pp. 335-354, 2011.

[13] R. K. Singh, S. N. Jena, S. Khan et al., "Development, crossspecies/genera transferability of novel EST-SSR markers and their utility in revealing population structure and genetic diversity in sugarcane," Gene, vol. 524, no. 2, pp. 309-329, 2013.

[14] F. M. You, N. Huo, Y. Q. Gu et al., "BatchPrimer3: a high throughput web application for PCR and sequencing primer design," BMC Bioinformatics, vol. 9, article 253, 2008.

[15] T.-W. Chen, R.-C. R. Gan, T. H. Wu et al., "FastAnnotator-an efficient transcript annotation web tool," BMC Genomics, vol. 13, no. 7, article S9, 2012.

[16] D. Hoisington, M. Khairallah, and D. González-de-León, Laboratory Protocols: CIMMYT Applied Molecular Genetics Laboratory, CIMMYT, Mexico DF, Mexico, 2nd edition, 1994.

[17] J. Sambrook and D. W. Russell, Molecular Cloning: A Laboratory Manual, Cold Spring Harbor Laboratory Press, Cold Spring Harbor, New York, NY, USA, 2001.

[18] A. Pavlíček, Š. Hrdá, and J. Flegr, "Free-tree-freeware program for construction of phylogenetic trees on the basis of distance data and bootstrap/jackknife analysis of the tree robustness. Application in the RAPD analysis of genus Frenkelia," Folia Biologica, vol. 45, no. 3, pp. 97-99, 1999.

[19] R. D. Page, “TreeView: an application to display phylogenetic trees on personal computers," Computer Applications in the Biosciences, vol. 12, no. 4, pp. 357-358, 1996.

[20] R. W. Mlchelmore, I. Paran, and R. V. Kesseli, "Identification of markers linked to disease-resistance genes by bulked segregant analysis: a rapid method to detect markers in specific genomic regions by using segregating populations," Proceedings of the National Academy of Sciences of the United States of America, vol. 88, no. 21, pp. 9828-9832, 1991.

[21] J. R. Ellis and J. M. Burke, "EST-SSRs as a resource for population genetic analyses," Heredity, vol. 99, no. 2, pp. 125132, 2007.

[22] J. Squirrell, P. M. Hollingsworth, M. Woodhead et al., "How much effort is required to isolate nuclear microsatellites from plants?” Molecular Ecology, vol. 12, no. 6, pp. 1339-1348, 2003. 
[23] C. Duran, N. Appleby, D. Edwards, and J. Batley, "Molecular genetic markers: discovery, applications, data storage and visualisation," Current Bioinformatics, vol. 4, no. 1, pp. 16-27, 2009.

[24] K. Kumari, M. Muthamilarasan, G. Misra et al., "Development of eSSR-markers in setaria italica and their applicability in studying genetic diversity, cross-transferability and comparative mapping in millet and non-millet species," PLoS ONE, vol. 8, no. 6, article e67742, 2013.

[25] M. Rossi, P. G. Araujo, F. Paulet et al., "Genomic distribution and characterization of EST-derived resistance gene analogs (RGAs) in sugarcane," Molecular Genetics and Genomics, vol. 269, no. 3, pp. 406-419, 2003.

[26] K. S. Aitken, P. A. Jackson, and C. L. McIntyre, "A combination of AFLP and SSR markers provides extensive map coverage and identification of homo(eo)logous linkage groups in a sugarcane cultivar," Theoretical and Applied Genetics, vol. 110, no. 5, pp. 789-801, 2005.

[27] G. M. Cordeiro, R. Casu, C. L. McIntyre, J. M. Manners, and R. J. Henry, "Microsatellite markers from sugarcane (Saccharum spp.) ESTs cross transferable to erianthus and sorghum," Plant Science, vol. 160, no. 6, pp. 1115-1123, 2001.

[28] L. R. Pinto, K. M. Oliveira, E. C. Ulian, A. A. F. Garcia, and A. P. De Souza, "Survey in the sugarcane expressed sequence tag database (SUCEST) for simple sequence repeats," Genome, vol. 47, no. 5, pp. 795-804, 2004.

[29] K. M. Oliveira, L. R. Pinto, T. G. Marconi et al., "Characterization of new polymorphic functional markers for sugarcane," Genome, vol. 52, no. 2, pp. 191-209, 2009.

[30] S. P. Kumpatla and S. Mukhopadhyay, "Mining and survey of simple sequence repeats in expressed sequence tags of dicotyledonous species," Genome, vol. 48, no. 6, pp. 985-998, 2005.

[31] R. K. Varshney, A. Graner, and M. E. Sorrells, "Genic microsatellite markers in plants: features and applications," Trends in Biotechnology, vol. 23, no. 1, pp. 48-55, 2005.

[32] E. Portis, I. Nagy, Z. Sasvári, A. Stágel, L. Barchi, and S. Lanteri, "The design of Capsicum spp. SSR assays via analysis of in silico DNA sequence, and their potential utility for genetic mapping," Plant Science, vol. 172, no. 3, pp. 640-648, 2007.

[33] B. T. James, C. Chen, A. Rudolph et al., "Development of microsatellite markers in autopolyploid sugarcane and comparative analysis of conserved microsatellites in sorghum and sugarcane," Molecular Breeding, vol. 30, no. 2, pp. 661-669, 2012.

[34] L. Qiu, C. Yang, B. Tian, J.-B. Yang, and A. Liu, "Exploiting EST databases for the development and characterization of ESTSSR markers in castor bean (Ricinus communis L.)," BMC Plant Biology, vol. 10, no. 1, article 278, 2010.

[35] F. C. Victoria, L. C. da Maia, and A. C. de Oliveira, "In silico comparative analysis of SSR markers in plants," BMC Plant Biology, vol. 11, article 15, 2011.

[36] M. Muthamilarasan, B. V. Suresh, G. Pandey, K. Kumari, S. K. Parida, and M. Prasad, "Development of 5123 intron-length polymorphic markers for large-scale genotyping applications in foxtail millet," DNA Research, vol. 21, no. 1, pp. 41-52, 2014.

[37] R. V. Kantety, M. La Rota, D. E. Matthews, and M. E. Sorrells, "Data mining for simple sequence repeats in expressed sequence tags from barley, maize, rice, sorghum and wheat," Plant Molecular Biology, vol. 48, no. 5-6, pp. 501-510, 2002.

[38] M. J. Lawson and L. Zhang, "Distinct patterns of SSR distribution in the Arabidopsis thaliana and rice genomes," Genome Biology, vol. 7, no. 2, article R14, 2006.
[39] L. C. Da Maia, V. Q. De Souza, M. M. Kopp, F. I. F. De Carvalho, and A. C. De Oliveira, "Tandem repeat distribution of gene transcripts in three plant families," Genetics and Molecular Biology, vol. 32, no. 4, pp. 822-833, 2009.

[40] I. Fabregat, K. S. Koch, T. Aoki et al., "Functional pleiotropy of an intramolecular triplex-forming fragment from the $3^{\prime}$-UTR of the rat Pigr gene," Physiological Genomics, vol. 5, no. 2, pp. 53-65, 2001.

[41] Y.-C. Li, A. B. Korol, T. Fahima, A. Beiles, and E. Nevo, "Microsatellites: genomic distribution, putative functions and mutational mechanisms: a review," Molecular Ecology, vol. 11, no. 12, pp. 2453-2465, 2002.

[42] J. R. Gatchel and H. Y. Zoghbi, "Diseases of unstable repeat expansion: mechanisms and common principles," Nature Reviews Genetics, vol. 6, no. 10, pp. 743-755, 2005.

[43] R. K. Mishra, B. H. Gangadhar, J. W. Yu, D. H. Kim, and S. W. Park, "Development and characterization of EST based SSR markers in Madagascar periwinkle (Catharanthus roseus) and their transferability in other medicinal plants," Plant Omics Journal, vol. 4, no. 3, pp. 154-162, 2011.

[44] R. K. Sharma, P. Bhardwaj, R. Negi, T. Mohapatra, and P. S. Ahuja, "Identification, characterization and utilization of unigene derived microsatellite markers in tea (Camellia sinensis L.)," BMC Plant Biology, vol. 9, no. 1, article 53, 2009.

[45] V. Pachauri, N. Taneja, P. Vikram, N. K. Singh, and S. Singh, "Molecular and morphological characterization of Indian farmers rice varieties (Oryza sativa L.)," Australian Journal of Crop Science, vol. 7, no. 7, pp. 923-932, 2013.

[46] B. Stich, A. E. Melchinger, M. Frisch, H. P. Maurer, M. Heckenberger, and J. C. Reif, "Linkage disequilibrium in European elite maize germplasm investigated with SSRs," Theoretical and Applied Genetics, vol. 111, no. 4, pp. 723-730, 2005.

[47] B. Hackauf and P. Wehling, "Identification of microsatellite polymorphisms in an expressed portion of the rye genome," Plant Breeding, vol. 121, no. 1, pp. 17-25, 2002.

[48] T. G. Marconi, E. A. Costa, H. R. Miranda et al., "Functional markers for gene mapping and genetic diversity studies in sugarcane," BMC Research Notes, vol. 4, no. 1, article 264, 2011.

[49] M. C. Saha, M. A. R. Mian, I. Eujayl, J. C. Zwonitzer, L. Wang, and G. D. May, "Tall fescue EST-SSR markers with transferability across several grass species," Theoretical and Applied Genetics, vol. 109, no. 4, pp. 783-791, 2004.

[50] S. Feingold, J. Lloyd, N. Norero, M. Bonierbale, and J. Lorenzen, "Mapping and characterization of new EST-derived microsatellites for potato (Solanum tuberosum L.)," Theoretical and Applied Genetics, vol. 111, no. 3, pp. 456-466, 2005.

[51] S. K. Parida, S. K. Kalia, S. Kaul et al., "Informative genomic microsatellite markers for efficient genotyping applications in sugarcane," Theoretical and Applied Genetics, vol. 118, no. 2, pp. 327-338, 2009.

[52] S. Gupta and M. Prasad, "Development and characterization of genic SSR markers in Medicago truncatula and their transferability in leguminous and non-leguminous species," Genome, vol. 52, no. 9, pp. 761-771, 2009.

[53] S. K. Gupta, R. Bansal, U. J. Vaidya, and T. Gopalakrishna, "Development of EST-derived microsatellite markers in mungbean [Vigna radiata (L.) Wilczek] and their transferability to other Vigna species," Indian Journal of Genetics and Plant Breeding, vol. 72, no. 4, pp. 468-471, 2012.

[54] M. S. Khan, S. Yadav, S. Srivastava, M. Swapna, A. Chandra, and R. K. Singh, "Development and utilisation of conserved-intron 
scanning marker in sugarcane," Australian Journal of Botany, vol. 59, no. 1, pp. 38-45, 2011.

[55] L. Y. Zhang, M. Bernard, P. Leroy, C. Feuillet, and P. Sourdille, "High transferability of bread wheat EST-derived SSRs to other cereals," Theoretical and Applied Genetics, vol. 111, no. 4, pp. 677687, 2005.

[56] A. Selvi, N. V. Nair, J. L. Noyer et al., "AFLP analysis of the phenetic organization and genetic diversity in the sugarcane complex, Saccharum and Erianthus," Genetic Resources and Crop Evolution, vol. 53, no. 4, pp. 831-842, 2006.

[57] M. Raghami, A. I. López-Sesé, M. R. Hasandokht, Z. Zamani, M. R. F. Moghadam, and A. Kashi, "Genetic diversity among melon accessions from Iran and their relationships with melon germplasm of diverse origins using microsatellite markers," Plant Systematics and Evolution, vol. 300, no. 1, pp. 139-151, 2014.

[58] B. W. S. Sobral and R. J. Honeycutt, "High output genetic mapping of polyploids using PCR-generated markers," Theoretical and Applied Genetics, vol. 86, no. 1, pp. 105-112, 1993.

[59] N. Msomi and F. Botha, "Identification of molecular markers linked to fibre using bulk segregant analysis," in Proceedings of the South African Sugar Technologists' Association (SASTA '94), vol. 68, pp. 41-45, 1994.

[60] L. Nivetha, N. Nair, P. Prathima, and A. Selvi, "Identification of microsatellite markers for high fibre content in sugarcane," Journal of Sugarcane Research, vol. 3, no. 1, pp. 14-19, 2013.

[61] C. M. Dussle, M. Quint, A. E. Melchinger, M. L. Xu, and T. Lübberstedt, "Saturation of two chromosome regions conferring resistance to SCMV with SSR and AFLP markers by targeted BSA," Theoretical and Applied Genetics, vol. 106, no. 3, pp. 485-493, 2003.

[62] C. Asnaghi, D. Roques, S. Ruffel et al., "Targeted mapping of a sugarcane rust resistance gene (Brul) using bulked segregant analysis and AFLP markers," Theoretical and Applied Genetics, vol. 108, no. 4, pp. 759-764, 2004.

[63] J. H. Daugrois, L. Grivet, D. Roques et al., "A putative major gene for rust resistance linked with a RFLP marker in sugarcane cultivar 'R570"' Theoretical and Applied Genetics, vol. 92, no. 8, pp. 1059-1064, 1996.

[64] C. T. Guimarães, G. R. Sills, and B. W. S. Sobral, "Comparative mapping of Andropogoneae: Saccharum L. (sugarcane) and its relation to sorghum and maize," Proceedings of the National Academy of Sciences of the United States of America, vol. 94, no. 26, pp. 14261-14266, 1997.

[65] R. Ming, S.-C. Liu, P. H. Moore, J. E. Irvine, and A. H. Paterson, "QTL analysis in a complex autopolyploid: genetic control of sugar content in sugarcane," Genome Research, vol. 11, no. 12, pp. 2075-2084, 2001.

[66] J. Guo, R. H. Y. Jiang, L. G. Kamphuis, and F. Govers, "A cDNA-AFLP based strategy to identify transcripts associated with avirulence in Phytophthora infestans," Fungal Genetics and Biology, vol. 43, no. 2, pp. 111-123, 2006.

[67] A. Fernández-del-Carmen, C. Celis-Gamboa, R. G. F. Visser, and C. W. B. Bachem, "Targeted transcript mapping for agronomic traits in potato," Journal of Experimental Botany, vol. 58, no. 11, pp. 2761-2774, 2007.

[68] B. Kloosterman, M. Oortwijn, J. uitdeWilligen et al., "From QTL to candidate gene: genetical genomics of simple and complex traits in potato using a pooling strategy," BMC Genomics, vol. 11, no. 1, article 158, 2010.

[69] X. Chen, P. E. Hedley, J. Morris, H. Liu, R. E. Niks, and N. R. Waugh, "Combining genetical genomics and bulked segregant analysis-based differential expression: an approach to gene localization," Theoretical and Applied Genetics, vol. 122, no. 7, pp. 1375-1383, 2011. 

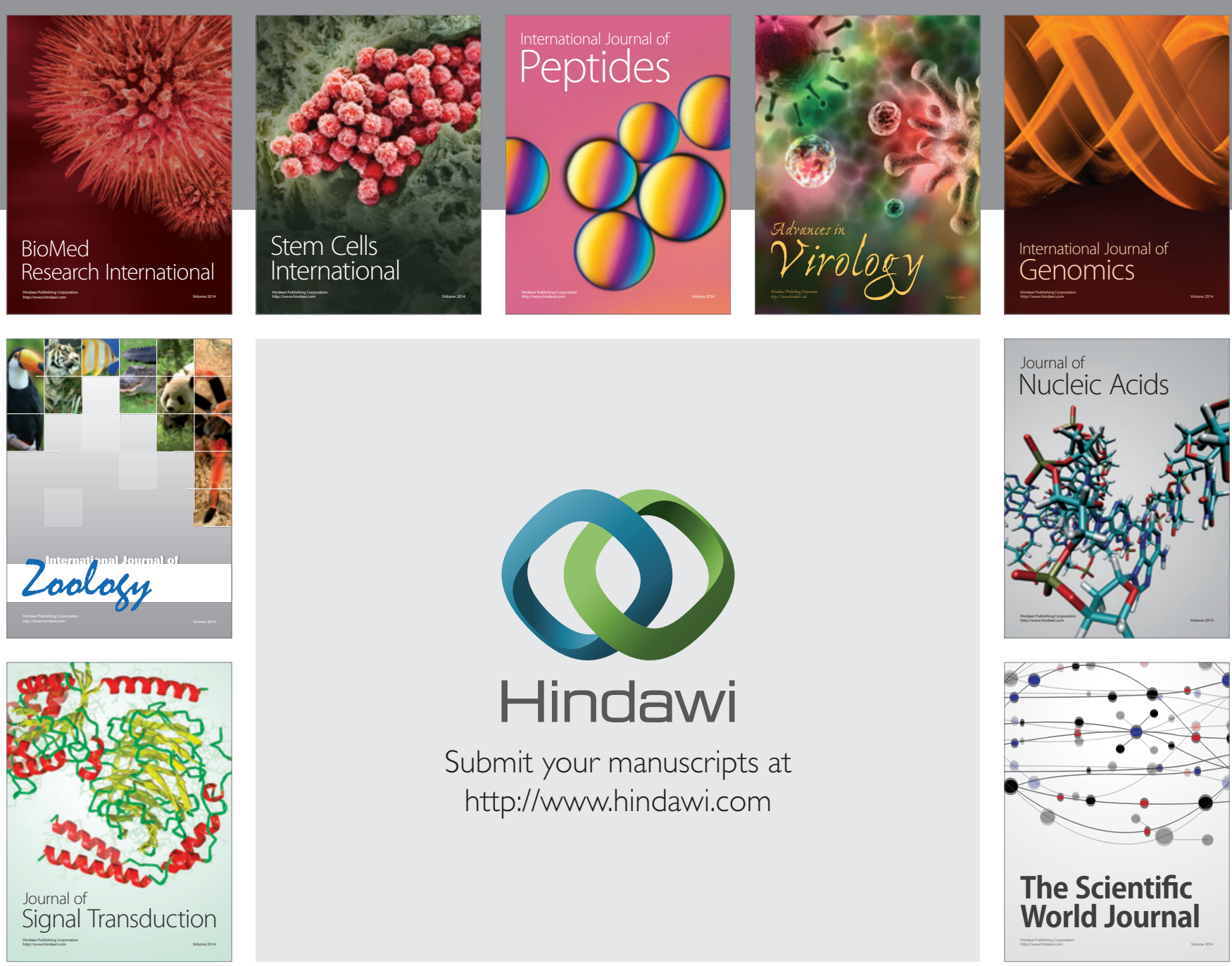

Submit your manuscripts at

http://www.hindawi.com
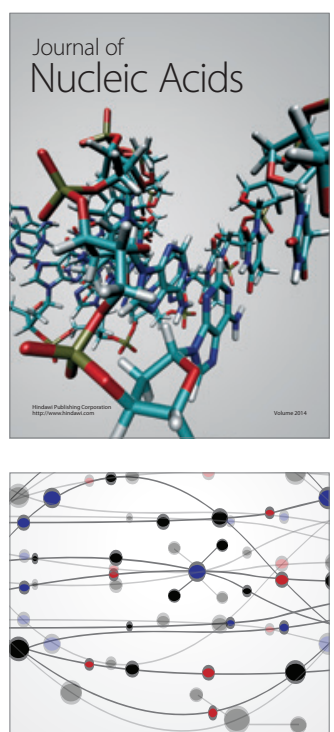

The Scientific World Journal
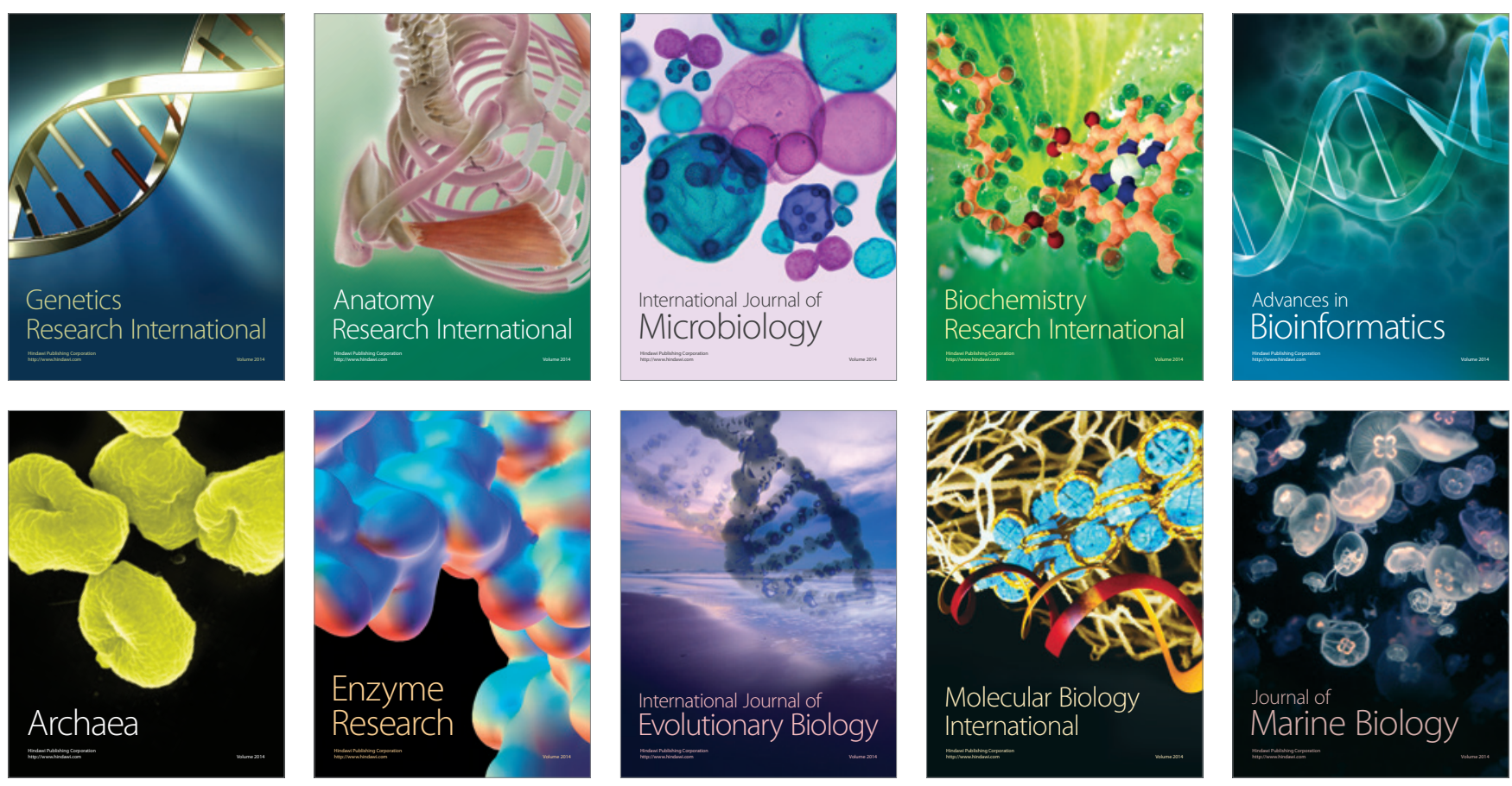